\title{
Measuring the Biphoton Temporal Wave Function with Polarization-Dependent and Time-Resolved Two-Photon Interference
}

\author{
Peng Chen, Chi Shu, Xianxin Guo, M. M. T. Loy, and Shengwang Du* \\ Department of Physics, The Hong Kong University of Science and Technology, Clear Water Bay, Kowloon, Hong Kong, China
}

(Received 19 September 2014; published 6 January 2015; corrected 8 January 2015)

\begin{abstract}
We propose and demonstrate an approach to measuring the biphoton temporal wave function with polarization-dependent and time-resolved two-photon interference. Through six sets of two-photon interference measurements projected onto different polarization subspaces, we can reconstruct the amplitude and phase functions of the biphoton temporal waveform. For the first time, we apply this technique to experimentally determine the temporal quantum states of the narrow-band biphotons generated from the spontaneous four-wave mixing in cold atoms.
\end{abstract}

DOI: 10.1103/PhysRevLett.114.010401

PACS numbers: 03.65.Wj, 03.67.Mn, 42.50.Dv

Photons are described by their discrete polarization states and field amplitude distribution in continuous time-space domains. The state density matrix in a discrete Hilbert space can be reconstructed using the well-developed quantum-state tomography [1-4]. To describe the temporal modes of photons, one needs a continuous-variable quantum-state tomography characterizing both the amplitude and the phase functions. Homodyne detection has been proven an efficient probe to characterize photonic (unentangled) Fock and coherent states [5-10]. However, most homodyne measurements of bipartite states have only aimed at verifying the entanglement [11-15]. A complete optical homodyne tomography for the time-frequency entangled two-photon (amplitude and phase) temporal waveform still remains a technical challenge [8].

There is an increasing interest in fully characterizing narrow-band biphotons because of their applications in realizing efficient light-matter quantum interfaces [16-19]. Using spontaneous four-wave mixing (SFWM) in cold atoms [20], the sub-MHz biphoton generation with a coherence time on the order of microseconds has been demonstrated [21-23]. Such a long coherence time of single photons allows manipulating their temporal waveforms [24-26] and their interaction with atoms in the time domain [27-29]. Owning to the nanosecond time resolution of commercially available single-photon counting modules (SPCM), the biphoton amplitude temporal profile can be directly measured from the coincidence counts. However, the coincidence counting does not distinguish between a time-frequency entangled state and a temporal-probability mixed state because it does not measure the phase distribution. Although additional evidences, such as the violation of the Cauchy-Schwarz inequality [30] and the antibunching of heralded single photons [31] can indicate the nonclassical properties, they do not provide a complete state information. It is believed that the time-frequency entanglement of biphotons generated from a continuouswave SFWM is naturally endowed by the energy conservation [32], but this claim has not been confirmed experimentally.

In this Letter, we propose and implement a technique to measure the temporal wave function of SFWM narrowband biphotons. Using six sets of symmetrized timeresolved two-photon interference measurements in different polarization bases, we reconstruct the biphoton temporal amplitude and phase functions.

The experimental setup for the narrow-band biphoton generation and the waveform measurement is shown in Fig. 1 . We work with right-angle SFWM in laser-cooled ${ }^{85} \mathrm{Rb}$ atoms loaded in a magneto-optical trap [33]. The energy difference of the two hyperfine ground states $|1\rangle=\mid 5 S_{1 / 2}$, $F=2\rangle$ and $|2\rangle=\left|5 S_{1 / 2}, F=3\right\rangle$ is $\hbar \Delta_{21}$, with $\Delta_{21}=$ $2 \pi \times 3036 \mathrm{MHz}$. The other two atomic energy levels are $|3\rangle=\left|5 P_{3 / 2}, F=3\right\rangle$ and $|4\rangle=\left|5 P_{3 / 2}, F=2\right\rangle$. The pump laser $\left(\omega_{p}\right)$ is far detuned from the transition $|1\rangle \rightarrow|3\rangle$ by $\Delta_{p}$, and the coupling laser $\left(\omega_{c}\right)$ is near resonance on the transition $|2\rangle \leftrightarrow|3\rangle$ (with an adjustable detuning $\Delta_{c}$ ). The Stokes $\left(\omega_{s}\right)$ and anti-Stokes $\left(\omega_{\text {as }}\right)$ photons are spontaneously generated from the transitions $|3\rangle \rightarrow|2\rangle$ and $|3\rangle \rightarrow|1\rangle$, respectively. The atomic optical depth is about 3 on the anti-Stokes transition. With linearly polarized, counterpropagating

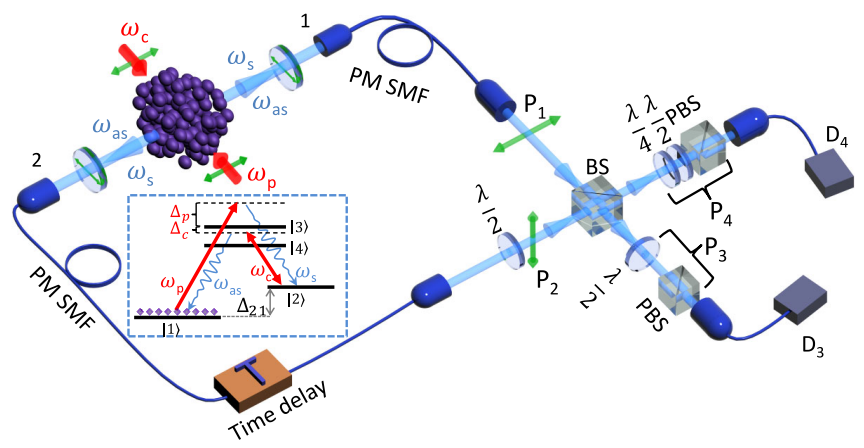

FIG. 1 (color online). Experimental setup for measuring the temporal wave function of narrow-band biphotons generated from backward-wave SFWM in the right-angle confirmation. 
pump and coupling laser beams, the phase-matched backward paired Stokes and anti-Stokes photons are collected at a right angle with respect to the pump-coupling direction. The single-spatial-mode biphoton temporal (complex) wave function at the source output is

$$
\begin{aligned}
\Psi\left(t_{s}, t_{\mathrm{as}}\right) & =\left\langle 0\left|\hat{a}_{s}\left(t_{s}\right) \hat{a}_{\mathrm{as}}\left(t_{\mathrm{as}}\right)\right| \Psi_{s, \mathrm{as}}\right\rangle \\
& =e^{-i \omega_{\mathrm{s} 0} t_{s}} e^{-i \omega_{\mathrm{as} 0} t_{\mathrm{as}}} \psi\left(t_{\mathrm{as}}-t_{s}\right),
\end{aligned}
$$

where $\omega_{s 0}$ and $\omega_{\text {as } 0}$ are the central optical angular frequencies of Stokes and anti-Stokes photons, respectively. The relative biphoton waveform $\psi\left(t_{\mathrm{as}}-t_{s}\right)$ can be written as

$$
\psi\left(t_{\mathrm{as}}-t_{s}\right)=A\left(t_{\mathrm{as}}-t_{s}\right) e^{i \phi\left(t_{\mathrm{as}}-t_{s}\right)},
$$

which is completely determined by the amplitude function $A\left(t_{\mathrm{as}}-t_{s}\right)$ and the phase function $\phi\left(t_{\mathrm{as}}-t_{s}\right)$. Because of the time ordering of the SFWM process and the slow-light effect on the anti-Stokes photons, $A$ is nonzero only at $t_{\mathrm{as}}-t_{s}>0$ [32]. That is, the anti-Stokes photon is always generated after its paired Stokes photon. The amplitude function can be determined from the two-photon coincidence measurement. In particular, the amplitude function is directly related to the Glauber correlation function $G^{(2)}\left(t_{\mathrm{as}}-t_{s}\right)=A^{2}\left(t_{\mathrm{as}}-t_{s}\right)$. The phase function $\phi\left(t_{\mathrm{as}}-t_{s}\right)$, as shown in this Letter, can be determined by the polarization-dependent two-photon interference.
With the right-angle SFWM geometry shown in Fig. 1, the photon pairs are spontaneously generated into two symmetric paths (1 and 2): the Stokes photons go to port 1 and the anti-Stokes photons to port 2, and vice versa. Therefore, there are Stokes and anti-Stokes photons in each output port. After passing through the horizontal linear polarizers, the photons are coupled into two polarization maintaining (PM) single-mode fibers (SMF). The outputs from the fibers are set to be horizontally $\left(P_{1} \leftrightarrow\right)$ and vertically $\left(P_{2} \uparrow\right.$, after a half-wave plate) polarized. The relative (optical) time delay $T$ between the two paths can be varied by setting the length difference of the PM SMFs. The photons are then combined together at a 50:50 beam splitter (BS), whose output ports 3 and 4 pass the polarizers $\left(P_{3}\right.$ and $\left.P_{4}\right)$ and are detected by two SPCMs $\left(D_{3}\right.$ and $\left.D_{4}\right)$. The coincidence counts between $D_{3}$ and $D_{4}$ are recorded with a time-bin width of $1 \mathrm{~ns}$. The linear polarizer $P_{3}$ consists of a half-wave plate and a polarizing beam splitter (PBS). The polarizer $P_{4}$ consists of a quarter-wave plate, a half-wave plate, and a PBS.

The two-frequency-mode field operators at the source outputs can be written as $\hat{a}_{1 \leftrightarrow}=\hat{a}_{1 s \leftrightarrow}+\hat{a}_{1 a s \leftrightarrow}$ and $\hat{a}_{2 \uparrow}=$ $\hat{a}_{2 s \uparrow}+\hat{a}_{2 a s \uparrow}$. The BS has the transformation $\hat{a}_{3}=\left(\hat{a}_{1 \leftrightarrow}-\right.$ $\left.i \hat{a}_{2 \uparrow}\right) / \sqrt{2}$ and $\hat{a}_{4}=\left(i \hat{a}_{1 \leftrightarrow}+\hat{a}_{2 \uparrow}\right) / \sqrt{2}$. The projection of the polarizers can be characterized as $\hat{a}_{P m}=\hat{a}_{m \leftrightarrow} \cos \alpha_{m}+$ $\hat{a}_{m \downarrow} \sin \alpha_{m} e^{i \theta_{m}} \quad(m=3,4)$. The two-photon relative wave function at the two detectors can be derived as [34]:

$$
\begin{aligned}
\psi_{34}(T, \tau)= & \frac{1}{2} \cos \alpha_{3} \sin \alpha_{4} e^{i \theta_{4}}\left[e^{-i \delta_{\omega}(\tau-T)} A(\tau-T) e^{i \phi(\tau-T)}+A(-\tau+T) e^{i \phi(T-\tau)}\right] \\
& +\frac{1}{2} \sin \alpha_{3} \cos \alpha_{4} e^{i \theta_{3}}\left[e^{i \delta_{\omega} T} A(-\tau-T) e^{i \phi(-\tau-T)}+e^{-i \delta_{\omega} \tau} A(\tau+T) e^{i \phi(\tau+T)}\right] .
\end{aligned}
$$

Here we define $\tau=t_{4}-t_{3}$ and $\delta_{\omega}=\omega_{a s 0}-\omega_{s 0}$. The additional phases $\delta_{\omega} T$ and $\delta_{\omega}(\tau-T)$ result from the central frequency difference $\delta_{\omega}$ between the paired photons and the relative time delay $T$. We can determine the Glauber correlation function as $G_{P 3 P 4}^{(2)}(T, \tau)=\left|\psi_{34}(T, \tau)\right|^{2}$. Using the two-photon detection efficiency $\eta$, the time-bin width $\Delta t$, and the total measurement time $\Delta t_{m}$, we can write the two-photon coincidence counts as $C_{P 3 P 4}(T, \tau)=$ $G_{P 3 P 4}^{(2)}(T, \tau) \eta \Delta t \Delta t_{m}$. From Eq. (3), coincidence measurements carry a combination of phase and amplitude information. Depending on the polarisers' settings, one can choose which information the measurement reveals. To retrieve the phase function $\phi(\tau)$, we measure six sets of coincidence counts under the conditions as shown in Table I and get [34]

$\cos [\Lambda(T, \tau)]=\frac{[B(T, \tau)+1]}{2 \sqrt{B(T, \tau)}} \frac{C_{\nearrow \nearrow}(T, \tau)-C_{\nearrow \searrow}(T, \tau)}{C_{\nearrow}(T, \tau)+C_{\nearrow \searrow}(T, \tau)}$, $\sin [\Lambda(T, \tau)]=\frac{[B(T, \tau)+1]}{-2 \sqrt{B(T, \tau)}} \frac{C_{\nearrow \circlearrowright}(T, \tau)-C_{\nearrow \circlearrowleft}(T, \tau)}{C_{\nearrow}(T, \tau)+C_{\nearrow \circlearrowleft}(T, \tau)}$,

where $B(T, \tau)=C_{\uparrow \leftrightarrow}(T, \tau) / C_{\leftrightarrow \uparrow}(T, \tau)$, and $\Lambda(T, \tau)=$ $\Xi(T, \tau)+\Lambda_{0} . \Lambda_{0}$ is the residual phase constant caused

TABLE I. Polarization projection configuration for the twophoton coincidence measurement after the beam splitter. $\uparrow$ Vertically polarized; $\leftrightarrow$ Horizontally polarized; $\nearrow 45^{\circ}$ linearly polarized; \ $-45^{\circ}$ linearly polarized; $\circlearrowright$ right-circularly polarized; $\circlearrowleft$ left-circularly polarized. For the optical polarizations, we take the convention where the wave is observed from the point of view of the source.

\begin{tabular}{cclc}
\hline \hline$\#$ & Coincidence counts & $P_{3}\left(\alpha_{3}, \theta_{3}\right)$ & $P_{4}\left(\alpha_{4}, \theta_{4}\right)$ \\
\hline 1 & $C_{\uparrow \leftrightarrow}(T, \tau)$ & $\uparrow(\pi / 2,0)$ & $\leftrightarrow(0,0)$ \\
2 & $C_{\leftrightarrow \uparrow}(T, \tau)$ & $\leftrightarrow(0,0)$ & $\uparrow(\pi / 2,0)$ \\
3 & $C_{\nearrow}(T, \tau)$ & $\nearrow(\pi / 4,0)$ & $\nearrow(\pi / 4,0)$ \\
4 & $C_{\nearrow}(T, \tau)$ & $\nearrow(\pi / 4,0)$ & $\searrow(-\pi / 4,0)$ \\
5 & $C_{\nearrow \circlearrowright}(T, \tau)$ & $\nearrow(\pi / 4,0)$ & $\circlearrowright(\pi / 4, \pi / 2)$ \\
6 & $C_{\nearrow \circlearrowleft}(T, \tau)$ & $\nearrow(\pi / 4,0)$ & $\circlearrowleft(\pi / 4,-\pi / 2)$ \\
\hline \hline
\end{tabular}


by imperfections of the optical components. The combined phase difference is defined as $\Xi(T, \tau)=\phi(\tau+T)-$ $\phi(\tau-T)-\delta_{\omega} T$ for $\tau>T$, and $\Xi(T, \tau)=\phi(-\tau-T)-$ $\phi(-\tau+T)+\delta_{\omega} T$ for $\tau<-T$. Because $\phi(\tau)=\phi(-\tau)$, there is $\Xi(T, \tau)=-\Xi(T,-\tau)$. The residual phase constant can be determined by $\left.\Lambda_{0}=\left(1 / 2 t_{c}\right) \int_{-t_{c}}^{t_{c}} \Lambda(T, \tau)\right] d \tau$ where $t_{c}$ is the temporal duration of the two-photon correlation function. Here we describe our algorithm for determining the phase function $(\tau>0)$. After obtaining $\Xi(T, \tau)$, we get $\phi(\tau+T)=\phi(\tau-T)+\delta_{\omega} T+\Xi(T, \tau)$. Setting a reference phase point at $\tau_{0}: \phi\left(\tau_{0}>0\right)=0$, we can obtain the phase function by the following recursion:

$$
\begin{aligned}
\phi\left(\tau_{0}+2 n T>T\right)= & \phi\left[\tau_{0}+2(n-1) T\right]+\delta_{\omega} T \\
& +\Xi\left[T, \tau_{0}+(2 n-1) T\right], \\
& (n= \pm 1, \pm 2, \ldots) .
\end{aligned}
$$

Similarly, one can get $\phi(\tau<-T)$. The temporal resolution is $2 T$.

There is a problem for directly applying Eq. (6) with a short $T$ to some special cases. For example, if the waveform has some nodes where the amplitude is zero, the phase at the amplitude nodes can take any value and thus is totally uncertain. In this case, the phase between the amplitude islands (the regions between amplitude nodes) is disconnected and their phase difference cannot be resolved when $T$ is too short. By taking a long $T$ we can bridge these amplitude islands and determine their phase difference but sacrifice the time resolution. To solve this problem, we propose a two-step recursion algorithm. First, we use a short $T_{s}$ to obtain a high resolution tomography inside each amplitude island. Next, we use a longer $T_{l}$ to measure the phase difference between the two islands without touching the nodes. The two-step algorithm allows us to obtain a full phase tomography with a resolution of $2 T_{s}$. In this Letter, we set $T_{s}=1.0 \mathrm{~ns}$ and $T_{l}=5.8 \mathrm{~ns}$.

We first perform a waveform measurement for degenerate biphotons $\left(\delta_{\omega}=0\right)$ by setting the parameters $\Delta_{p}=\Delta_{21}-2 \pi \times 3 \mathrm{MHz}$ and $\Delta_{c}=-2 \pi \times 10 \mathrm{MHz}$. The pump and coupling lasers Rabi frequencies are $\Omega_{p}=$ $2 \pi \times 164.3 \mathrm{MHz}$ and $\Omega_{c}=2 \pi \times 39.2 \mathrm{MHz}$, respectively. The additional pump frequency detuning $\Delta_{p}-\Delta_{21}=$ $-2 \pi \times 3 \mathrm{MHz}$ is for compensating the frequency shift caused by the ac Stark effect $\Omega_{p}^{2} /\left(4 \Delta_{p}\right)=$ $2 \pi \times 2.2 \mathrm{MHz}$ and the residual magnetic-field-induced Zeeman shift of the ground level $|1\rangle$. With these parameters, theory predicts that the biphoton waveform displays a damped Rabi oscillation with $\pi$-phase flip across the amplitude nodes [32]. The two-photon coincidence counts $C_{12}(\tau) \propto A^{2}(\tau)+A^{2}(-\tau)$ are shown in Fig. 2(a), where the plot at $\tau>0$ is the Stokes to anti-Stokes correlation $\left[A^{2}(\tau)\right]$ and that at $\tau<0$ is the anti-Stokes to Stokes correlation $\left[A^{2}(-\tau)\right]$. The measured combined phase-difference function excluding the residual phase constant (for $T_{l}=5.8 \mathrm{~ns}$ )

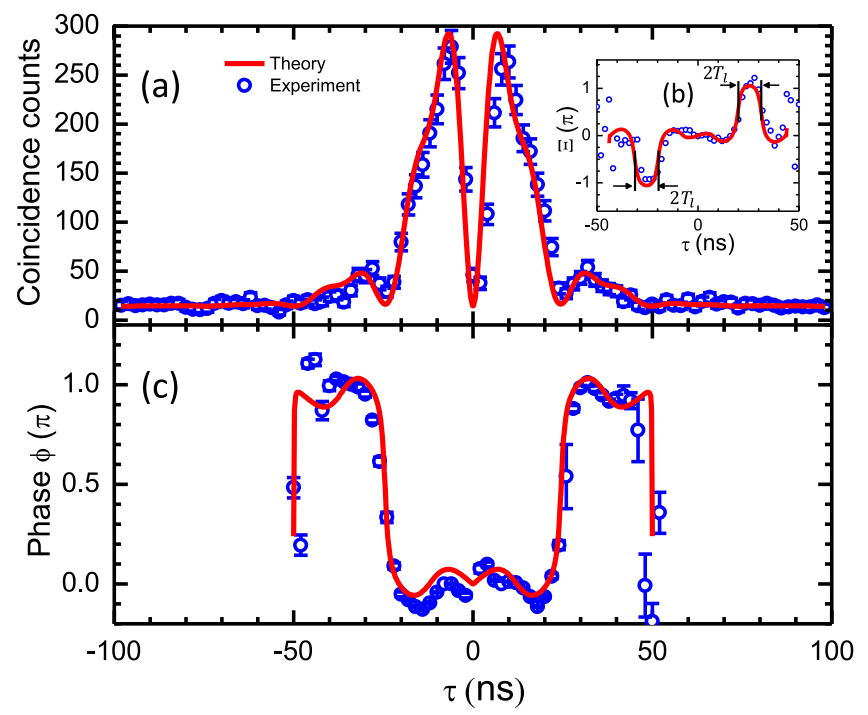

FIG. 2 (color online). Degenerate biphoton $\left(\delta_{\omega}=0\right)$ waveform with Rabi oscillation. (a) Two-photon coincidence counts before the BS. (b) The measured combined phase-difference function $\Xi(\tau)$ for $T_{l}=5.8 \mathrm{~ns}$. (c) The reconstructed phase $\phi(\tau)$.

is shown in Fig. 2(b). Together with the data for $T_{s}=1.0 \mathrm{~ns}$, we reconstruct the phase function $\phi(\tau)$ shown in Fig. 2(c), which clearly shows $\pi$-phase jumps across the amplitude nodes at $\tau= \pm 25$ and $\pm 50 \mathrm{~ns}$. From the phase jumps we determine the Rabi oscillation period of $25 \mathrm{~ns}$, which corresponds to an oscillation angular frequency of $2 \pi \times 40 \mathrm{MHz}$. It agrees with the theoretical effective coupling Rabi frequency $\sqrt{\Omega_{c}^{2}+\Delta_{c}^{2}}=2 \pi \times 40.5 \mathrm{MHz}$. We obtain the solid curves following the theory in the interaction picture [32] that accounts for the effect of the atomic energy level $|4\rangle$ on the SFWM process. The experimental result agrees with the theoretical prediction within the temporal correlation time of about $50 \mathrm{~ns}$. The phase data points near $\tau=50 \mathrm{~ns}$ have large deviations because the amplitude is damped to near zero and the phase becomes largely uncertain. We do not plot the reconstructed phase points and the theoretical phase curve at $|\tau|>50 \mathrm{~ns}$ where the amplitude approaches zero.

We next demonstrate that our method can be used to measure the complex waveform of nondegenerate biphotons $\left(\delta_{\omega}=2 \pi \times 43 \mathrm{MHz}\right)$ by setting the parameters $\Delta_{p}=\Delta_{21}+2 \pi \times 40 \mathrm{MHz}, \Delta_{c}=-2 \pi \times 10 \mathrm{MHz}, \Omega_{p}=$ $2 \pi \times 164.3 \mathrm{MHz}$, and $\Omega_{c}=2 \pi \times 42.6 \mathrm{MHz}$. The measured $\Xi$ is shown in Fig. 3(b), where the phase-difference jump $\left(2 \delta T_{l}\right)$ across $\tau=0$ is caused by the product of the frequency difference $\delta_{\omega}$ and the time delay $T_{l}$ as predicted from Eq. (6). The reconstructed phase function and the theoretical plot are shown in Fig. 3(c) and agree well with each other.

Last, we show that our technique can also be used to measure the biphoton waveform without amplitude nodes. We reduce the coupling laser detuning to zero $\left(\Delta_{c}=0\right)$ and lower the coupling laser power $\left(\Omega_{c}=2 \pi \times 19.6 \mathrm{MHz}\right)$ 


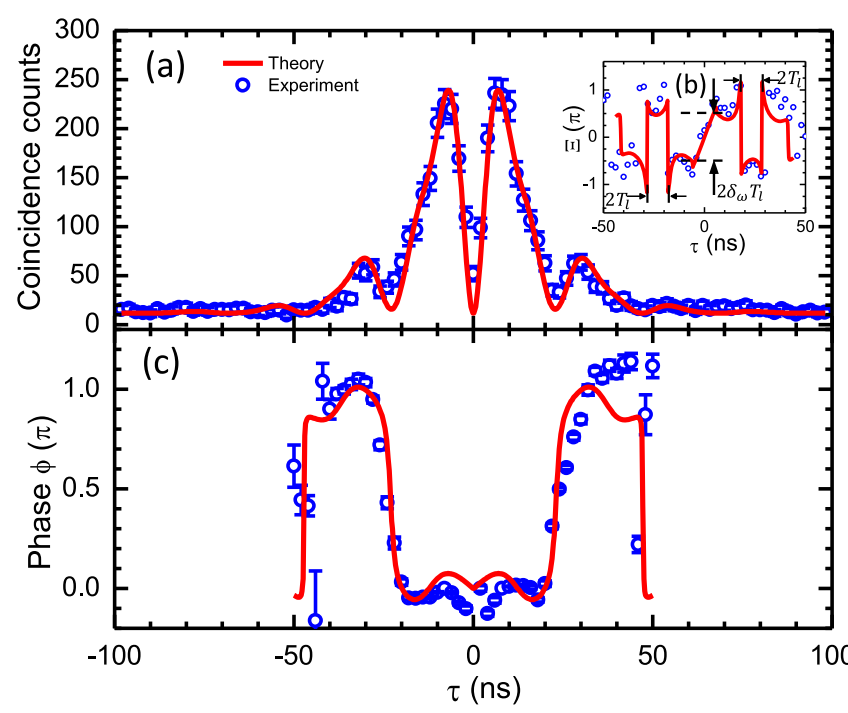

FIG. 3 (color online). Nondegenerate biphoton $\left(\delta_{\omega}=\right.$ $2 \pi \times 43 \mathrm{MHz}$ ) waveform with Rabi oscillation. (a) Two-photon coincidence counts before the BS. (b) The measured combined phase-difference function $\Xi(\tau)$ for $T_{l}=5.8 \mathrm{~ns}$. (c) The reconstructed phase $\phi(\tau)$.

to prolong the biphoton correlation time. As shown in Fig. 4(a), the second oscillation period is completely damped out. The measured combined phase-difference function is shown in Fig. 4(b), whose step change $\left(2 \delta_{\omega} T_{l}\right)$ near $\tau=0$ can be used to resolve the frequency difference $\delta_{\omega}$ between the paired photons. The reconstructed phase function is shown in Fig. 4(c) and agrees well with the theory.

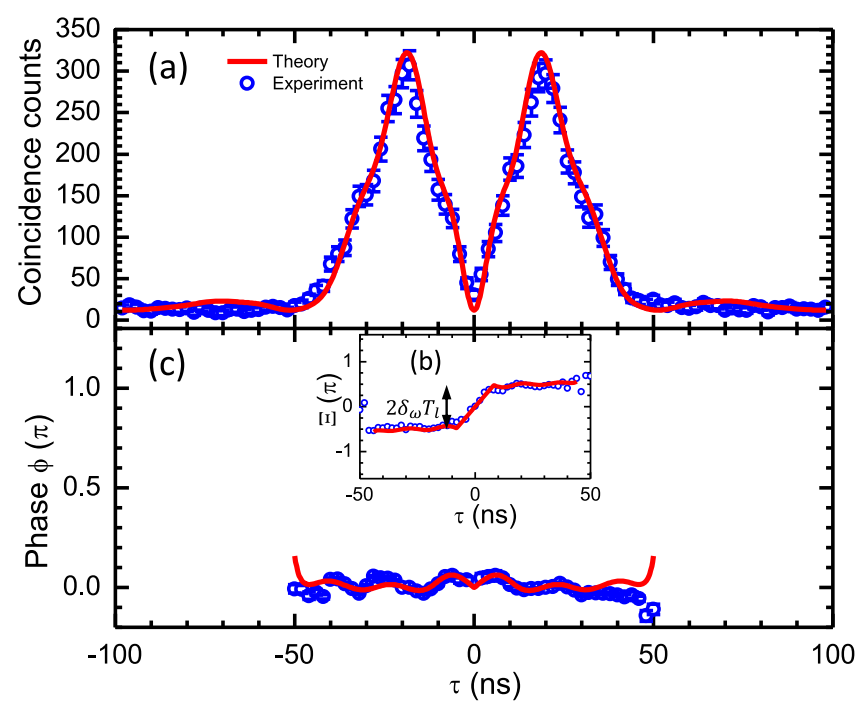

FIG. 4 (color online). Nondegenerate biphoton $\left(\delta_{\omega}=\right.$ $2 \pi \times 43 \mathrm{MHz}$ ) waveform with overdamped Rabi oscillation. (a) Two-photon coincidence counts before the BS. (b) The measured combined phase-difference function $\Xi(\tau)$ for $T_{l}=5.8$ ns. (c) The reconstructed phase $\phi(\tau)$.
Furthermore, we measure the nonclassical properties of the photon source by verifying its violation of the Cauchy-Schwarz inequality [30]. Given the normalized cross-correlation function $g_{s, \text { as }}^{(2)}(\tau)$ and the autocorrelation functions $g_{s, s}^{(2)}(\tau)$ and $g_{\text {as,as }}^{(2)}(\tau)$, a classical source fulfills the inequality $\mathrm{CS}=\left[g_{s, \text { as }}^{(2)}(\tau)\right]^{2} /\left[g_{s, s}^{(2)}(0) g_{\text {as,as }}^{(2)}(0)\right] \leq 1$. The violation of the Cauchy-Schwarz inequality in a quantum light source is associated with the nonexistence of a positive Glauber-Sudarshan $P$ function [35]. The biphoton source can be used to produce heralded single photons. We confirm this by measuring the antibunching effect in the autocorrelation function $g_{c}^{(2)}$ of the heralded photons [31]. A classical source always displays the bunching effect $g_{c}^{(2)} \geq 1$. A two-photon Fock state gives $g_{c}^{(2)}=0.5$ [36]. The measured $\mathrm{CS}_{\max }$ and $g_{c}^{(2)}$ of our biphoton source are shown in Table II. In all cases, the Cauchy-Schwarz inequality is violated, and $g_{c}^{(2)}$ is well below the twophoton threshold of 0.5 .

In summary, we present a technique to measure the temporal wave function of narrow-band biphotons generated from SFWM in cold atoms. In all three degenerate and nondegenerate cases, our experimental results agree with the theoretical predictions. For the first time, we experimentally resolve the $\pi$-phase jumps in the Rabi oscillations. The degeneracy of the photon frequencies does not need to be preassumed and the frequency difference can be determined from the measured $\Xi(T, \tau)$. We find that the major systematic errors are caused by the imperfection from the BS and polarizers. We attribute the disagreement between the experimental data and the theoretical curves, especially at those amplitude nodes, to the presence of multiple Zeeman substates which are not taken into account by the simple SFWM theoretical model [32]. From the determined amplitude and phase functions, we obtain that the full widths at half maximum of the biphoton power spectra for Figs. 2, 3, and 4 are 52, 47, and $22 \mathrm{MHz}$ from their respective Fourier transforms. With the experimentally determined amplitude $A(\tau)$ and phase $\phi(\tau)$ functions, we can reconstruct the biphoton wave function $\Psi\left(t_{s}, t_{\mathrm{as}}\right)=$ $e^{-i \omega_{s 0} t_{s}} e^{-i \omega_{\mathrm{as} 0} t_{\mathrm{as}}} A\left(t_{\mathrm{as}}-t_{s}\right) e^{i \phi\left(t_{\mathrm{as}}-t_{s}\right)}$ following Eqs. (1) and (2). Because the two-photon complex waveform cannot be expressed as a product state in the time domain, we verify the time (frequency) entanglement of the biphoton source.

TABLE II. Violation of Cauchy-Schwarz inequality and the measured conditional $g_{c}^{(2)}$. $\mathrm{CS}_{\max }$ is measured at the peak of the two-photon correlation function. $g_{c}^{(2)}$ is integrated over the entire waveform temporal length of $50 \mathrm{~ns}$.

\begin{tabular}{lrc}
\hline \hline & \multicolumn{1}{c}{$\mathrm{CS}_{\max }$} & $g_{c}^{(2)}$ \\
\hline Fig. 2 & $204 \pm 30$ & $0.27 \pm 0.05$ \\
Fig. 3 & $89 \pm 13$ & $0.16 \pm 0.07$ \\
Fig. 4 & $203 \pm 22$ & $0.19 \pm 0.02$ \\
\hline \hline
\end{tabular}


Although in the above demonstration we take advantage of the right-angle geometry, our technique can be extended to more general cases. The key is to have the Stokes and anti-Stokes photons sharing the same spatial modes. For example, in the case where the Stokes and anti-Stokes photons are generated with a small angle to the pumpcoupling direction [21-23], we can follow the modified configuration shown in the Supplemental Material [34], where the phase-matched photon pairs are produced into two symmetric paths and counteroverlapped by two mirrors. This method can also be applied to narrow-band biphotons generated from cavity-enhanced spontaneous parametric down-conversion [17].

The work was supported by the Hong Kong Research Grants Council (Project No. 601113). C. S. acknowledges support from the Undergraduate Research Opportunities Program at the Hong Kong University of Science and Technology.

Note added.-We became aware that during the review process a work of measuring the biphoton temporal wave function using a homodyne method was published [37]. Our method of polarization-dependent and time-resolved two-photon interference is different and does not require any external reference.

*dusw@ust.hk

[1] A. G. White, D. F. V. James, P. H. Eberhard, and P. G. Kwiat, Phys. Rev. Lett. 83, 3103 (1999).

[2] D. F. V. James, P. G. Kwiat, W. J. Munro, and A. G. White, Phys. Rev. A 64, 052312 (2001).

[3] R. B. A. Adamson and A. M. Steinberg, Phys. Rev. Lett. 105, 030406 (2010).

[4] C. Bernhard, B. Bessire, T. Feurer, and A. Stefanov, Phys. Rev. A 88, 032322 (2013).

[5] A. I. Lvovsky, H. Hansen, T. Aichele, O. Benson, J. Mlynek, and S. Schiller, Phys. Rev. Lett. 87, 050402 (2001).

[6] A. Ourjoumtsev, R. Tualle-Brouri, and P. Grangier, Phys. Rev. Lett. 96, 213601 (2006).

[7] A. MacRae, T. Brannan, R. Achal, and A. I. Lvovsky, Phys. Rev. Lett. 109, 033601 (2012).

[8] A. I. Lvovsky and M. G. Raymer, Rev. Mod. Phys. 81, 299 (2009).

[9] O. Morin, C. Fabre, and J. Laurat, Phys. Rev. Lett. 111, 213602 (2013).

[10] Z. Qin, A. S. Prasad, T. Brannan, A. MacRae, A. Lezama, and A. I. Lvovsky, arXiv:1405.6251.

[11] Z. Y. Ou, S. F. Pereira, H. J. Kimble, and K. C. Peng, Phys. Rev. Lett. 68, 3663 (1992).

[12] C. Schori, J. L. Sørensen, and E. S. Polzik, Phys. Rev. A 66, 033802 (2002).
[13] J. Laurat, G. Keller, J. A. Oliveira-Huguenin, C. Fabre, T. Coudreau, A. Serafini, G. Adesso, and F. Illuminati, J. Opt. B 7, S577 (2005).

[14] M. Vasilyev, S.-K. Choi, P. Kumar, and G. Mauro D'Ariano, Phys. Rev. Lett. 84, 2354 (2000).

[15] V. D’Auria, S. Fornaro, A. Porzio, S. Solimeno, S. Olivares, and M. G. A. Paris, Phys. Rev. Lett. 102, 020502 (2009).

[16] J. K. Thompson, J. Simon, H. Loh, and V. Vuletic, Science 313, 74 (2006).

[17] X. H. Bao, Y. Qian, J. Yang, H. Zhang, Z.-B. Chen, T. Yang, and J.-W. Pan, Phys. Rev. Lett. 101, 190501 (2008).

[18] M. Scholz, L. Koch, and O. Benson, Phys. Rev. Lett. 102, 063603 (2009).

[19] J. Fekete, D. Rielander, M. Cristiani, and H. de Riedmatten, Phys. Rev. Lett. 110, 220502 (2013).

[20] V. Balic, D. A. Braje, P. Kolchin, G. Y. Yin, and S. E. Harris, Phys. Rev. Lett. 94, 183601 (2005).

[21] S. Du, P. Kolchin, C. Belthangady, G. Y. Yin, and S. E. Harris, Phys. Rev. Lett. 100, 183603 (2008).

[22] K. Liao, H. Yan, J. He, S. Du, Z.-M. Zhang, and S.-L. Zhu, Phys. Rev. Lett. 112, 243602 (2014).

[23] L. Zhao, X. Guo, C. Liu, Y. Sun, M. M. T. Loy, and S. Du, Optica 1, 84 (2014).

[24] P. Kolchin, C. Belthangady, S. Du, G. Y. Yin, and S. E. Harris, Phys. Rev. Lett. 101, 103601 (2008).

[25] S. Du, J. Wen, and C. Belthangady, Phys. Rev. A 79, 043811 (2009).

[26] J. F. Chen, S. Zhang, H. Yan, M. M. T. Loy, G. K. L. Wong, and S. Du, Phys. Rev. Lett. 104, 183604 (2010).

[27] S. Zhang, J. F. Chen, C. Liu, M. M. T. Loy, G. K. L. Wong, and S. Du, Phys. Rev. Lett. 106, 243602 (2011).

[28] S. Zhou, S. Zhang, C. Liu, J. F. Chen, J. Wen, M. M. T. Loy, G. K. L. Wong, and S. Du, Opt. Express 20, 24124 (2012).

[29] S. Zhang, C. Liu, S. Zhou, C.-S. Chuu, M. M. T. Loy, and S. Du, Phys. Rev. Lett. 109, 263601 (2012).

[30] J. F. Clauser, Phys. Rev. D 9, 853 (1974).

[31] P. Grangier, G. Roger, and A. Aspect, Europhys. Lett. 1, 173 (1986).

[32] S. Du, J. Wen, and M. H. Rubin, J. Opt. Soc. Am. B 25, C98 (2008).

[33] C. Liu, J. F. Chen, S. Zhang, S. Zhou, Y.-H. Kim, M. M. T. Loy, G. K. L. Wong, and S. Du, Phys. Rev. A 85, 021803(R) (2012).

[34] See the Supplemental Material at http://link.aps.org/ supplemental/10.1103/PhysRevLett.114.010401 for a detailed theoretical derivation and the modified measurement schematics for narrow-band biphotons generated from a backward-wave SFWM without the right-angle geometry.

[35] M. D. Reid and D.F. Walls, Phys. Rev. A 34, 1260 (1986).

[36] R. Loudon, The Quantum Theory of Light, 3rd ed. (Oxford University Press, New York, 2000).

[37] F. A. Beduini, J. A. Zielinska, V. G. Lucivero, Y. A. de Icaza Astiz, and M. W. Mitchell, Phys. Rev. Lett. 113, 183602 (2014). 